\title{
Maryvale Hospital to Close
}

Abrazo Health Care has announced that it intends to close Maryvale Hospital effective December 18, 2017. Maryvale Hospital has had declining admissions and was realigned as a satellite facility of Abrazo West Campus in Goodyear in May 2017. Abrazo said they hoped to place most of the 300 Maryvale employees at other Abrazo medical centers.

Richard A. Robbins, MD

Editor, SWJPCC 